Original Research Article

\title{
A prospective study comparing the safety and efficacy of combination of topical tacrolimus $0.1 \%$ and mometasone furoate $0.01 \%$ with topical tacrolimus $0.1 \%$ alone in vitiligo
}

\author{
Febina C. Puthiyapurayil ${ }^{1 *}$, Anuradha Mothalampet ${ }^{1}$, Najeeba Riyas ${ }^{2}$
}

\begin{abstract}
${ }^{1}$ Department of Pharmacology, ${ }^{2}$ Department of Dermatology and Venereology, Government Medical College, Kozhikode, Kerala, India
\end{abstract}

Received: 02 June 2017

Accepted: 27 June 2017

\section{*Correspondence to:}

Dr. Febina C. Puthiyapurayil, Email: febinacp@gmail.com

Copyright: (C) the author(s), publisher and licensee Medip Academy. This is an openaccess article distributed under the terms of the Creative Commons Attribution NonCommercial License, which permits unrestricted noncommercial use, distribution, and reproduction in any medium, provided the original work is properly cited.

\begin{abstract}
Background: Vitiligo is a common acquired skin disorder. Corticosteroids are considered to be the first line treatment for vitiligo, but have side effects on prolonged use. Calcineurin inhibitors like Tacrolimus are found to be effective which is used either alone or in combination with steroids. The advantage of combination therapy of Tacrolimus with Mometasone is that it provides additional benefit for the disease as well as the adverse effects of steroids can be minimised. The main objective of this study is to compare the safety and efficacy of combination of topical Tacrolimus $0.1 \%$ and Mometasone furoate $0.01 \%$ with topical Tacrolimus $0.1 \%$ alone in vitiligo.

Methods: In this study 90 patients were divided into two groups to receive either combination treatment of topical Tacrolimus $0.1 \%$ and Mometasone furoate $0.01 \%$ or topical Tacrolimus $0.1 \%$ alone. The efficacy was measured in terms of mean improvement in VASI (Vitiligo Area Scoring Index) scores at 2weeks, 4 weeks, 6weeks, 12 weeks, and 24weeks from the start of therapy. Safety of the drugs were measured in terms of adverse effects reported during therapy.

Results: At the start of the study, mean VASI scores of both groups were comparable. At the endpoint, mean VASI improvement score was statistically significant among the combination group (57.82vs31.45, p <0.001). Adverse effects were found to be less in combined treatment group $(\mathrm{p}=0.046)$.

Conclusions: Combination treatment (Tacrolimus+ Mometasone) found to be superior to Tacrolimus alone in the treatment of vitiligo in terms of efficacy and safety.
\end{abstract}

Keywords: Mometasone furoate, Tacrolimus, Vitiligo

\section{INTRODUCTION}

Vitiligo is a one of the common often heritable acquired disorder characterised by well circumscribed milky white cutaneous macules or patches devoid of identifiable melanocytes. It occurs worldwide, with a prevalence of 0.1 percent to 2.0 percent. ${ }^{1,2}$ Of this $0.45 \%$ occur in Indians. The pattern of this disorder differs in different countries and in various locations in the same country.

The incidence of vitiligo in persons with racially pigmented skins is higher, although reliable figures are not available. Increased incidence is reported among female patients based on outpatient attendances, but the frequency in the population is the same in both sexes. ${ }^{3}$ Between $30 \%$ and $40 \%$ of patients have a positive family history, and a genetic factor is undoubtedly involved. ${ }^{4}$

Different modalities of treatment have been used in vitiligo like phototherapy with psoralens, steroids, heliotherapy, lasers, vitamin D analogues and skin grafting. As the pathogenesis of this disease is still obscure, the treatment of vitiligo has generally been unsatisfactory and often disappointing. 
For the past decades, monotherapy with topical steroids has been the most common treatment for vitiligo in children and adults. Topical tacrolimus also has shown promising results in the treatment of vitiligo in various studies. But only very few studies are available to evaluate the effectiveness of the combination, topical tacrolimus and mometasone in the treatment of vitiligo. Hence the aim of this study is to evaluate efficacy and safety of combined therapy of tacrolimus and mometasone over that of monotherapy with Tacrolimus alone in treatment of vitiligo.

\section{METHODS}

This study was designed as a prospective comparative study. The study was conducted in department of Dermatology and venereology, Medical College, Kozhikode and Department of Pharmacology at Medical College, Kozhikode.

The study was done after getting the clearance and under the vigilant guidance of the Institutional Research Committee and Institutional Ethics Committee, Government Medical College, Kozhikode. It was conducted over a period of one year from January 2014 to January 2015.

A total of 90 patients who fulfilled the inclusion criteria were enrolled in the study. They were of age $>18$ years, either sex with stable vitiligo involving $<30 \%$ of body surface area. Patients showed no evidence of spontaneous regimentation. They received no treatment for the last one month.

The patients with lip-tip type of vitiligo or mucosal involvement and those who had known hypersensitivity to tacrolimus or mometasone were excluded. Patients who had history of autoimmune disease, impaired liver or renal function, hypercalcemia, photosensitivity, hypertension, cardiovascular disease, pregnancy, lactation, concomitant use of vitamin $\mathrm{D}$, calcium and any other drug that can affect calcium homeostasis were also excluded.

Half of the patients were given a combination treatment i.e. topical Mometasone furoate $0.01 \%$ applied in the day time and topical Tacrolimus $0.1 \%$ applied in the night. The other half given only topical Tacrolimus $0.1 \%$ in the night.

Written informed consent was taken from all patients. All the relevant details regarding history, examination, treatment, type of vitiligo, sites of involvement and extent of the disease were recorded on a proforma.

Vitiligo area scoring index(VASI) is used as a measure to assess disease severity and outcome of treatment. ${ }^{5,6}$ Total body VASI calculated using formula:

VASI $=\sum[$ Hand units $] \times$ Residual depigmentation
One hand unit, which encompasses the palm plus the volar surface of all the digits, is approximately $1 \%$ of the total body surface area and is used as a guide to estimate the baseline percentage of vitiligo involvement in each body region. The body is divided into five separate and mutually exclusive regions: hands, upper extremities (excluding hands), trunk, lower extremities (excluding feet), and feet. The axillary region is included with the upper extremities while the buttocks and inguinal areas are included with the lower extremities.

The extent of residual depigmentation is expressed by the following percentages: $0,10 \%, 25 \%, 50 \%, 75 \%, 90 \%$, or $100 \%$. At $100 \%$ depigmentation, no pigment is present; at $90 \%$, specks of pigment are present; at $75 \%$, the depigmented area exceeds the pigmented area; at $50 \%$, the depigmented and pigmented areas are equal; at $25 \%$, the pigmented area exceeds the depigmented area; at $10 \%$, only specks of depigmentation are present.

Subjects in both groups were treated and studied for 6 months. Efficacy assessment was made by assessing the VASI score recorded at 2 weeks, 4 weeks, 6 weeks, 12 weeks and 24 weeks. The improvement in VASI score is assessed by comparing the VASI scores at the start of study to the scores at the end of study. These scores correlate directly with the outcome of treatment. The degree of repigmentation in vitiligo following treatment is also assessed by VASI scoring system. Improvement in repigmentation is indicated by improvement in VASI score which is graded in table1:

\section{Table 1: Grading of repigmentation in vitiligo using VASI score.}

\begin{tabular}{|lll|}
\hline Grades & Percentage & $\begin{array}{l}\text { Degree of } \\
\text { repigmentation }\end{array}$ \\
\hline Grade 0 & $0 \%$ & Absent \\
\hline Grade I & $1 \%-25 \%$ & Slight \\
\hline Grade II & $26 \%-50 \%$ & Moderate \\
\hline Grade III & $51 \%-75 \%$ & Good \\
\hline Grade IV & $76 \%-100 \%$ & Excellent \\
\hline
\end{tabular}

Adverse drug reactions were monitored during the study period based on history, observation, and self reported adverse drug reactions at each review. Patients were asked to report immediately if they experienced any untoward side effects.

\section{RESULTS}

Statistical analysis was done using Statistical package for social service (SPSS) software. Patients who completed the entire 6 months of treatment period were included in the statistical analysis.

Gender distribution of the two groups were compared using Chi-square test. Efficacy comparison of the study drugs were made in terms of improvement in 
repigmentation using VASI scoring system and was compared using independent sample t test.

Safety profile was assessed in terms of self reported adverse effects as well as through clinical examination in both the groups. The adverse effect profile was analysed using Chi-Square test. $\mathrm{p}$ value of less than 0.05 was considered to be statistically significant.

One patient from each group was lost to follow up during the course of study. Rest of the patients completed the treatment period of 24 weeks.

Percentage of depigmentation in these vitiligo patients were assessed with VASI scoring system at the beginning of study. Patients were then followed up at 2 weeks, 4 weeks, 6 weeks, 12 weeks and 24 weeks.

Baseline parameters like age, sex is comparable for both the groups. Distribution of patients according to age of onset of vitiligo shows that maximum patients $(\mathrm{N}=42)$ belong to the age group 26-35 years.

Regarding gender, the percentage of female patients was found to be higher than the male patients in both the groups. This is consistent with the study conducted by Kovacs who referred to a preponderance of females among patients with vitiligo.

Of the total 90 patients with vitiligo, family history was given by 5 patients $(5.6 \%)$.

The degree of repigmentation in vitiligo following treatment is also assessed by VASI scoring system. Improvement in repigmentation is indicated by improvement in VASI score which is then graded from grade 0 to grade IV.

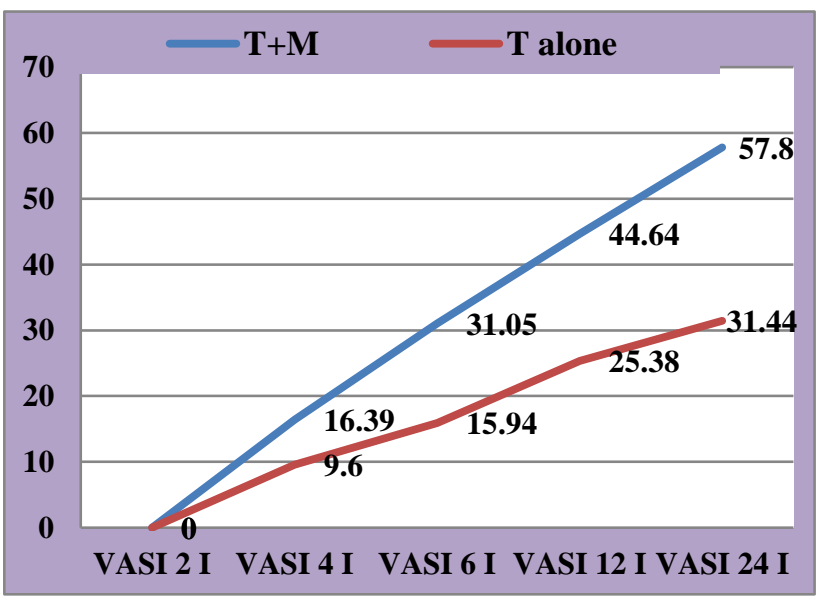

Figure 1: Mean VASI improvement score of the study groups from the start of the study to the end point.

At the start of the study, baseline VASI score of combination group $(\mathrm{T}+\mathrm{M})$ was $99 \pm 50.49$ and that of the Tacrolimus alone group $113 \pm 59.73$ and these baseline values are comparable $(p=0.238)$. In both groups, improvement is not seen till 4 weeks. At the end of the study there was significant improvement in mean VASI score in each treatment group. But when comparing both groups, combination group shows better improvement (57.8 \pm 22.4$)$ when compared to Tacrolimus alone group $(31.4 \pm 28.57)$ and the results were significant $(\mathrm{p}<0.001)$.

Final mean VASI improvement score (VASI 24 I) in each group was graded from grade 0 to grade IV. It is seen that all the patients responded in combination group $(\mathrm{T}+\mathrm{M})$. Excellent repigmentation $(76-100 \%)$ was observed in $36.4 \%$ cases. One patient showed complete repigmentation with the combination treatment as shown in Figure 2.

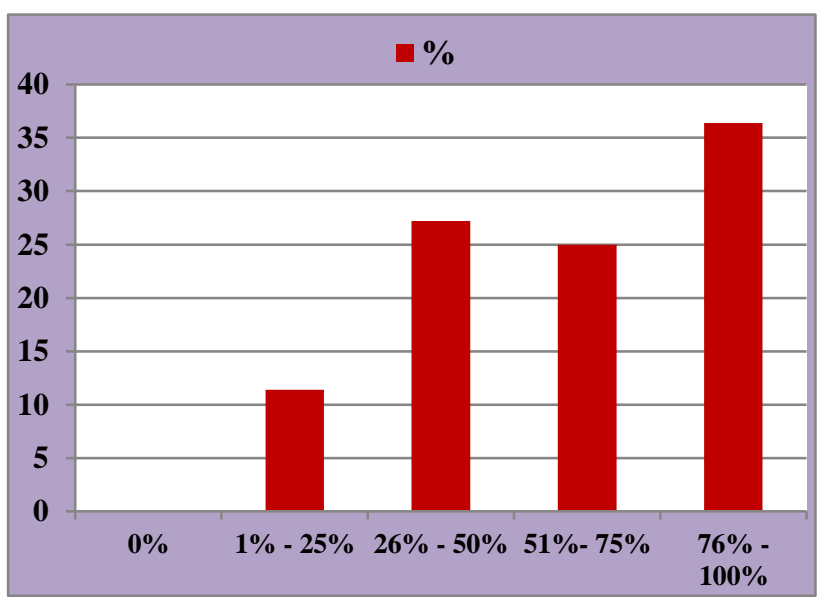

Figure 2: Grading of repigmentation among combination group.

In the Tacrolimus alone group, 17 cases $(38.3 \%)$ did not show any improvement at all. Excellent repigmentation observed in $9.2 \%$ cases, with one patient showing complete repigmentation as shown in Figure 3.

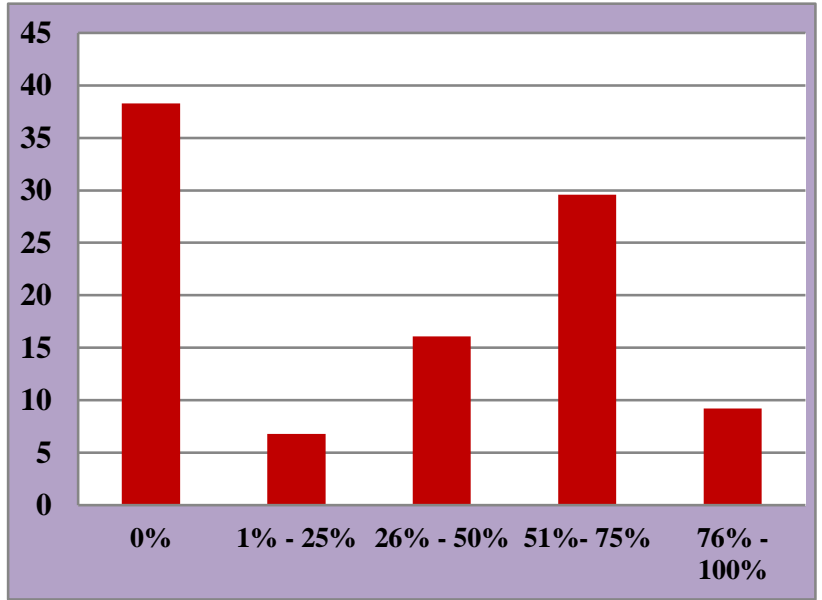

Figure 3: Grading of repigmentation in tacrolimus alone group.

Mean values of the VASI improvement Scores are compared for both the groups from the start of the study to 
the endpoint. Both the groups showed an increase in VASI score improvement as the study progressed. Combination treatment $(\mathrm{T}+\mathrm{M})$ group showed better improvement in mean VASI scores when compared to Tacrolimus ( $\mathrm{T}$ alone) group which is depicted in Figure 1.

In the combination group, $9.1 \%$ of patients reported adverse effects whereas in Tacrolimus alone group, 36.4\% of patients reported adverse effects. Only reported adverse effect in combination group was burning sensation. In the other group, $20.5 \%$ of patients reported burning sensation. Other adverse effects in this group were soreness, itching. In one patient of Tacrolimus alone group, contact dermatitis was reported.

\section{DISCUSSION}

Despite significant advances made in the past few years, treatment of vitiligo still remains a challenge. Different treatment modalities have been tried for this disease but results are not satisfactory. Relative failure to achieve desired effects with existing therapies in this cosmetically disfiguring disease has always stimulated research. Several treatment modalities with varying success rates are currently in use.

In vitiligo involving less than $10 \%$ body surface area, the most common therapy used is topical steroid. But the problem with the use of steroid in chronic disorders like vitiligo are the adverse effects produced by prolonged use such as atrophy and telengiectasias which are more common with Class 1 (very potent) topical steroids. Mometasone furoate is a nonfluorinated Class IV corticosteroid with a good safety profile. it is only half as potent in suppressing the hypothalamic-pituitary-adrenal axis as compared to very potent steroids and produces limited local and systemic adverse effects. Mometasone alone has been proven to be effective in the treatment of vitiligo with minimal side effects even in children. ${ }^{7}$ The range of response has been between $20 \%$ and $90 \%$ improvement, usually not a complete cure. Adverse effects and poor efficacy have led to the search for new alternatives.

Topical calcineurin inhibitors have been an interesting and innovative addition to the armamentarium of topical drugs used for vitiligo and they offer many advantages over corticosteroids for the management of chronic skin disorders in which prolonged treatment periods are needed. Among topical calcineurin inhibitors, Tacrolimus as well as Pimecrolimus have been used for a long time in the treatment of vitiligo. Topical Tacrolimus is derived from the bacteria Streptomyces tsukubaensis. Since 2002, several studies have shown this agent to have promising results in vitiligo. The response rates vary between $63 \%$ and $89 \%$ depending on the type and location of vitiligo, with good results for face and neck lesions. ${ }^{8-10}$ Topical Tacrolimus alone has been proven to be effective in treatment of vitiligo in different age groups in different studies conducted in different parts of the world. Even in patients with generalized vitiligo it has found to be effective in combination with NBUVB therapy. Topical Tacrolimus has been shown to be as effective as topical Clobetasol propionate in a study conducted in children.

In the studies where only topical calcineurin inhibitors have been used, some percentage of patients did not show any repigmentation even after 12 weeks of treatment. In our study the advantage of adding topical Mometasone to topical Tacrolimus is that at the end of 12 weeks all the patients showed some degree of repigmentation and this is very important for the patient satisfaction.

\section{Limitations}

Limitations of study included small sample size, non availability of treatment modalities to non affordable patients and usage of drugs in stable vitiligo involving head and neck region.

\section{ACKNOWLEDGEMENTS}

Author would like to thank the guide Dr. Anuradha M., Professor and Head of the Department of Pharmacology, Govt. Medical College, Kozhikode, who with her able guidance, optimistic attitude and constant encouragement gave all the confidence and determination to complete this dissertation.

Also would like to express the deepest sense of gratitude and thanks to Dr. Najeeba Riyas, Professor and Head of the Department of Dermatology and Venereology Govt. Medical College, Kozhikode for her able guidance and support during the study.

Authors will always remember with gratitude all the patients who willingly participated in this study.

Funding: No funding sources

Conflict of interest: None declared

Ethical approval: The study was approved by the Institutional Ethics Committee

\section{REFERENCES}

1. Kovacs SO. Vitiligo. J Am Acad Dermatol. 1998 May;38:647-66.

2. Halder RM, Young CM. New and emerging therapies for vitiligo. Dermatol Clin. 2000;18:79-88.

3. Howitz J, Brodthagen H, Schwartz M, Thomsen K. Prevalence of vitiligo. Epidemiological survey on the Isle of Bornholm, Denmark. Arch Dermatol. 1977;113:47-52.

4. Lerner AB. On the etiology of vitiligo and gray hair. Am J Med. 1971 Aug;51(2):141-7.

5. Hamzavi I, Jain H, McLean D, Shapiro J, Zeng H, Lui H. Parametric modeling of narrowband UV-B phototherapy for vitiligo using a novel quantitative tool: The Vitiligo Area Scoring Index. Arch Dermatol. 2004 Jun;140(6):677-83. 
6. Rossiter ND, Chapman P, Haywood IA. How big is a hand? Burns. 1996;22(3):230-1.

7. Köse O1, Arca E, Kurumlu Z. J Dermatolog Treat. Mometasone cream versus pimecrolimus cream for the treatment of childhood localized vitiligo. 2010 May;21(3):133-9.

8. Silverberg NB, Lin P, Travis L, Farley-Li J, Mancini AJ, Wagner AM, et al. Tacrolimus ointment promotes repigmentation of vitiligo in children: a review of 57 cases. J Am Acad Dermatol. 2004;51:760-6.

9. Hartmann A, Bröcker EB, Hamm H. Occlusive treatment enhances efficacy of tacrolimus $0.1 \%$ ointment in adult patients with vitiligo: results of a placebo-controlled 12-month prospective study. Acta Derm Venereol. 2008;88(5):474-9.

10. Fai D, Cassano N, Vena GA. Narrow-band UVB phototherapy combined with tacrolimus ointment in vitiligo: a review of 110 patients. J Eur Acad Dermatol Venereol. 2007 Aug;21(7):916-20.

Cite this article as: Puthiyapurayil FC, Mothalampet A, Riyas N. A prospective study comparing the safety and efficacy of combination of topical tacrolimus $0.1 \%$ and mometasone furoate $0.01 \%$ with topical tacrolimus $0.1 \%$ alone in vitiligo. Int $\mathrm{J}$ Basic Clin Pharmacol 2017;6:2005-9. 